\title{
A battle in the brain: Post-traumatic stress disorder checklist is an effective screening tools needed for post-traumatic stress disorder in primary care
}

\author{
Sarah Piekarski
}

\section{University of Ottawa}

Post-traumatic stress disorder (PTSD) is a debilitating psychological condition that affects an estimated ten percent of the Canadian population. ${ }^{1}$ Affecting all ages, PTSD develops from exposure to a traumatic event with the threat of death or severe bodily harm, accompanied by feelings of intense fear and horror. ${ }^{2}$ PTSD can manifest years later, and often without warning - for this reason, PTSD presents a difficult diagnosis and one that is often missed by primary health care physicians. ${ }^{3}$ A quick, accurate and comprehensive screening tool for PTSD, such as the PCL-Civilian, ${ }^{4}$ needs to be regularly implemented in the Canadian primary health care assessment of individuals with extreme anxiety or depression that could be an underlying PTSD issue.

With the string of recent suicides in Canadian Military personnel in 2013, and with more PTSD related suicides projected to occur within the next year, a greater awareness of the presenting symptoms of PTSD as well as better screening methods need to be in place in Canadian primary care settings. ${ }^{5}$ The post-traumatic stress disorder checklist, PCL-Civilian, is one possible screening tool currently available that can be used to accurately assess the possibility of a PTSD diagnosis in a timely manner. The PCL-C can be facilitated by the physician or taken alone by the patient, making it particularly useful in a primary care context when the administration of a structured interview is not suitable. The PCL-C is based upon the PTSD symptoms in the Diagnostic and Statistical Manual of Mental Disorders (DSM)-Fourth Edition. Items are based upon a five-point Likert scale, and are added to obtain a total severity score; the greater the score, the more symptoms present. A cutoff score of fifty is commonly used and has been shown to be a good indicator of PTSD when compared to the Clinician Administered PTSD Scale (CAPS) and the Structured Clinical Interview for DSM (SCID). ${ }^{6}$ The PCL has shown a strong correlation of 0.79 with the CAPS and 0.90 with the Mississippi PTSD Scale; ${ }^{7}$ in addition, the current gold standard for PTSD diagnosis, the CAPS, is time-consuming to administer, thus making the faster PCL-Civilian a more appropriate primary care tool. By determining a correct diagnosis of PTSD earlier, treatment could be easier, and recovery quicker. While the $\mathrm{PCL}$-Civilian has a tendency to overestimate the prevalence of PTSD, employing the quick, seventeen-question PCL-Civilian for the symptoms of PTSD will aid in the identification of the disorder and subsequently the referral to appropriate treatment. A timely diagnosis of PTSD can aid in the avoidance of maladaptive behaviours such as alcohol and substance abuse, ${ }^{8}$ as well as family anxiety, and personal strife. Therefore, it follows from this evidence that the sooner PTSD is correctly identified, the sooner the individual can be referred to behavioural health specialists and support groups to minimize negative outcomes.

The PCL-Civilian screening tool simplifies the diagnosis and screening of those with suspected PTSD. For soldiers returning from combat, a severe traumatic brain injury presents symptoms almost indistinguishable from PTSD. ${ }^{8}$ In addition, misdiagnosing PTSD as depression or anxiety can lead to improper treatment. Medicating PTSD has not been demonstrated to improve the individual's functioning and recovery - as is common in anxiety and mood disorders - as rapidly as behavioural treatment. ${ }^{9}$ The diagnosis of PTSD is further complicated by individuals that may have a co-morbid condition of an anxiety mood disorder, which may mask the underlying diagnosis of PTSD from the practitioner. Unless the patient volunteers personal information regarding their trauma, it is difficult to identify PTSD without a solid framework - the PCL-Civilian can provide that framework. With the assistance of the PCL-Civilian screening tool, the complexity in making an appropriate PTSD preliminary diagnosis can be lessened for the primary health care professional. Through the adoption of the PCL-Civilian, Canadian primary care providers can $>$ 
screen individuals for PTSD with a high degree of temporal stability, internal consistency, test-retest reliability, and convergent validity. ${ }^{7,10}$

Overall, health care investments into the development and implementation of a more effective PTSD screening test, like the PCL-Civilian, into Canadian primary care are needed. Greater awareness of the diagnosis of PTSD by primary health care professionals could lead to the more aggressive treatment of PTSD and overall improved functioning of those individuals. The PCL-Civilian screening tool could benefit primary health care practitioners by lessening the length of consultation times and improving a physician's confidence in diagnosis. The tool will aid in identifying those with PTSD and will alleviate the complexity involved in a diagnosis. Screening implementation with the PCL may reduce misdiagnoses and lead to more appropriate healthcare utilization. The PCL screening tool can thus be used to assist primary care providers in the assessment of a PTSD diagnosis and to monitor patients' symptom changes before, during, and after treatment.

\section{References}

1. Van Ameringen, M., Mancini, C., Mancini, C., Patterson, B., Boyle, M. PostTraumatic Stress Disorder in Canada. CNS Neuroscience \& Therapeutics. 2008; 14:171-181.

2. American Psychiatric Association. Diagnostic and Statistical Manual of Mental Disorders, (5th ed.). Arlington, Va.: American Psychiatric Publishing. 2013

3. Liebschutz, J., Saitz, R., Brower, V., Keane, T.M., Lloyd-Travaglini, C., Averbuch, T., Samet, J.H. PTSD in Urban Primary Care: High Prevalence and Low Physician Recognition. Society of General Internal Medicine. 2007, June; 22(6):719-726.

4. Ruggiero, K.J., Del Ben, K., Scotti, J.R., Rabalais, A.E. Pychometric Properties of the PTSD Checklist - Civilian Version. Journal of Traumatic Stress. 2003; 16: 495-502.

5. Magruder, K.M., Frueh, B.C., Knapp, R.G., Davis, L., Hamner, M.B., Herbert Martin, R., Gold, P.B., Arana, G.W. Prevalence of posttraumatic stress disorder in Veterans Affairs primary care clinics. General Hospital Psychiatry. 2005, May; 27(3): 169-179.

6. Gravely, A.A., Cutting, A., Nugent, S., Grill, J., Carlson, K., Spoont, M. Validity of PTSD diagnoses in VA administration data: Comparison of VA administration PTSD diagnoses to self-reported PTSD Checklist scores. Journal of Rehabilitation Research \& Development. 2011; 48(1): 21-30.

7. Wilkins, K.C., Lang, A.J., Norman, S.B. Synthesis of the Psychometric Properties of the PTSD Checklist (PCL) Military, Civilian, and Specific Version. Depress Anxiety. 2011 July; 28(7): 596-606.

8. Williamson, J.B., Heilman, K.M., Porges, E.C., Lamb, D.G., Porges, S.W. A possible mechanism for PTSD symptoms in patients with traumatic brain injury: central automatic network disruption. Frontiers in neuroengineering. 2013, Dec 19; 6:13.

9. Wilkins, K., Lang, A.J., Norman, S.B. Synthesis of the Psychometric Properties of the PTSD Checklist (PCL) Military, Civilian, and Specific Versions. Depress Anxiety. 2011, July; 28(7): 596-606

10. Blanchard, E.B., Jones-Alexander, J, Buckley, T.C., Forneris, C.A. Psychometric properties of the PTSD checklist (PCL). Behavioural Research and Therapy. 1996; 34: 669-673.

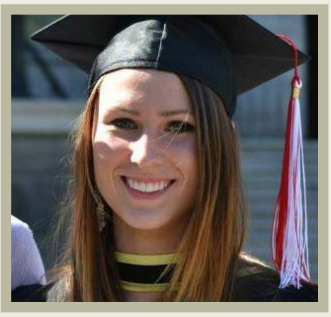

\section{Sarah Piekarski}

Sarah Piekarski is a Masters student at the University of Ottawa, working to supplement her undergraduate Bachelor of Science degree from McGill, with a focused study in Interdisciplinary Health Sciences. She is conducting her research on cognition and navigation, and plans to further her education with a degree in Rehabilitation Sciences. 